\title{
Nanostructured Lipid Carriers (NLC) as Vehicles for Topical Administration of Sesamol: In Vitro Percutaneous Absorption Study and Evaluation of Antioxidant Activity
}

\section{Authors}

Carmelo Puglia ${ }^{1}$, Maria Rosaria Lauro ${ }^{2}$, Alessia Offerta ${ }^{1}$, Lucia Crasci $^{1}$, Lucia Micicchè $^{1}$, Anna Maria Panico ${ }^{1}$, Francesco Bonina ${ }^{1}$, Giovanni

Puglisi ${ }^{1}$

\section{Affiliations}

1 Department of Drug Sciences, University of Catania, Catania, Italy

2 Department of Pharmacy, University of Salerno, Fisciano (SA), Italy

Key words

Sesamum indicum, Pedaliaceae, NLC, in vitro percutaneous absorption, Franz cells, DSC, ORAC assay

$\begin{array}{ll}\text { received } & \text { October 29, 2015 } \\ \text { revised } & \text { February 1, 2016 } \\ \text { accepted } & \text { March 10, 2016 }\end{array}$

Bibliography

DOI http://dx.doi.org/10.1055/s-0042-105293

Published online April 28, 2016 | Planta Med 2017; 83: 398-404

(c) Georg Thieme Verlag KG Stuttgart · New York I ISSN 0032-0943

Correspondence

Prof. Carmelo Puglia

Department of Drug Sciences, University of Catania

Viale Andrea Doria 6, 95125 Catania, Italy

Phone: + 390957384209 , Fax: + 39095222239

capuglia@unict.it

\section{ABSTRACT}

Sesamol is a natural phenolic compound extracted from Sesamum indicum seed oil. Sesamol is endowed with several beneficial effects, but its use as a topical agent is strongly compromised by unfavorable chemical-physical properties. Therefore, to improve its characteristics, the aim of the present work was the formulation of nanostructured lipid carriers as drug delivery systems for topical administration of sesamol.

Two different nanostructured lipid carrier systems have been produced based on the same solid lipid (Compritol ${ }^{\circledR} 888$ ATO) but in a mixture with two different kinds of oil phase such as Miglyol ${ }^{\circledR} 812$ (nanostructured lipid carrier-M) and sesame oil (nanostructured lipid carrier-PLUS). Morphology and dimensional distribution of nanostructured lipid carriers have been characterized by differential scanning calorimetry and photon correlation spectroscopy, respectively. The release pattern of sesamol from nanostructured lipid carriers was evaluated in vitro determining drug percutaneous absorption through excised human skin. Furthermore, an oxygen radical absorbance capacity assay was used to determine their antioxidant activity.

From the results obtained, the method used to formulate nanostructured lipid carriers led to a homogeneous dispersion of particles in a nanometric range. Sesamol has been encapsulated efficiently in both nanostructured lipid carriers, with higher encapsulation efficiency values $(>90 \%$ ) when sesame oil was used as the oil phase (nanostructured lipid carrier-PLUS).

In vitro evidences show that nanostructured lipid carrier dispersions were able to control the rate of sesamol diffusion through the skin, with respect to the reference formulations.

Furthermore, the oxygen radical absorbance capacity assay pointed out an interesting and prolonged antioxidant activity of sesamol, especially when vehiculated by nanostructured lipid carrier-PLUS.

$\begin{array}{ll}\text { ABBREVIATIONS } \\ \text { AAPH } & \text { 2,2'-azobis(2-amidinopropane) dihydrochloride } \\ \text { DSC } & \text { differential scanning calorimetry } \\ \text { EE } & \text { encapsulation efficiency } \\ \text { FL } & \text { fluorescein } \\ \text { HSH } & \text { high shear homogenization } \\ \text { NLCS } & \text { nanostructured lipid carriers } \\ \text { ORAC } & \text { oxygen radical absorbance capacity } \\ \text { SCE } & \text { stratum corneum epidermis } \\ \text { SES } & \text { sesamol } \\ \text { SLNs } & \text { solid lipid nanoparticles } \\ \text { US } & \text { ultrasound }\end{array}$

\section{Introduction}

Plant-derived active constituents are gaining importance in today's quest for potent and efficacious antioxidant compounds because of their natural background and lesser toxicity compared to synthetic molecules. These actives are widely used to formulate both cosmetic and pharmaceutical products intended for topical application.

SES (5-hydroxy-1,3-benzodioxole or 3,4-methylenedioxyphenol) is a natural phenolic compound extracted from Sesamum indicum L. (Pedaliaceae) seed oil ( $\bullet$ Fig. 1). Several beneficial effects of SES were reported including antioxidation, chemoprevention, antimutagenic, and hepatotoxic properties [1,2]. These effects were associated with its hydroxyl radical scavenging and in- 


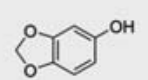

- Fig. 1 Chemical structure of sesamol.

hibitory activities of lipid peroxidation, although the biological functions of SES have not entirely been elucidated yet [3].

Cosmetic use of topical SES is also of interest as this agent is included among the ingredients of anti-wrinkle and antiaging formulations [4]. Recently, it has been used to formulate skinwhitening products in virtue of a high anti-tyrosinase activity compared to popular compounds such as kojic acid and $\beta$-arbutin $[5,6]$.

Notwithstanding these interesting features, SES clinical application is limited because of its physicochemical properties. SES shows a water solubility of $38 \pm 1.20 \mathrm{mg} / \mathrm{mL}$ and a log $P$ value of $1.29 \pm 0.01$, therefore after topical application, it passes quickly through all the skin layers with a quick passage into systemic circulation. To overcome these difficulties, this study is proposing an innovative approach: the delivery of SES formulated in NLCS, which is suitable for topical administration.

NLCs are innovative lipid nanoparticles composed of a binary mixture of a solid lipid and a liquid lipid and show increased drug loading compared with SLNs, the first generation of lipid nanoparticles [7]. Furthermore, NLCs minimize other limits of SLNs such as the drug expulsion phenomena and the excessive particle dilution in the aqueous dispersion (from $1 \%$ to $30 \%$ ) [8]. NLCs are investigated for different routes of administration with a more recent interest for topical application [9-11].

Therefore, in this study, we have evaluated the potential use of NLCs for topical administration of SES and the carrier ability to increase the in vitro antioxidant efficacy of this compound. With this aim, SES loaded NLCs were prepared and characterized on their physicochemical properties and by the DSC technique to obtain information regarding the nanostructure of the lipid matrix. Furthermore, in vitro percutaneous absorption studies were affected using excised human skin membranes (i.e., SCE) to evaluate the amount of SES permeated through the skin once released from the lipid nanocarriers. Finally, SES loaded NLCs were studied in order to understand the influence of the vehicle on SES antioxidant properties using an in vitro ORAC assay.

\section{Results and Discussion}

In this study, we evaluated two NLC formulations obtained using two different oil components, Miglyol 812 (NLC-M) and sesame oil (NLC-PLUS). It is widely reported that the inclusion of oil is useful in the optimization of NLC formulation and to hold higher amounts of a drug. In fact, the oil, behaving as an impurity in the solid lipid, reduces the particle crystallinity, conferring better stability and a higher suitability for the controlled release $[7,12,13]$. In the present work, we decided to use sesame oil as the oil phase of NLCs to study a possible synergism between this natural ingredient and SES.

Sesame oil is an edible vegetable oil derived from sesame seeds. It is composed of a high amount of fatty acids such as linoleic acid ( $41 \%$ of total), oleic acid (39\%), palmitic acid ( $8 \%)$, and stearic acid (5\%) and furthermore it contains important active compounds, such as lignans and tocopherols, which are responsible for the outstanding antioxidant activity of sesame oil. Recent studies have pointed out that the synergistic activities of these active components, instead of the activity of each one alone, could well explain the bioactivity of this oil [14-17]. Therefore, the vehicle, called NLC-PLUS, was formulated to be an upgrade of the conventional NLC system, able, on the one hand, to strengthen the antioxidant efficacy of SES, in virtue of the pool of antioxidant substances contained in the sesame oil, and on the other hand, to optimize its percutaneous absorption by exploiting the technological strategy represented by the SES encapsulation in NLCs.

Both NLCs and SLNs were prepared by coupling HSH with a US treatment. This method has many advantages compared to preparations performed using only one of these techniques, such as high homogeneity and reduced dimensions of nanoparticle dispersions [18].

As reported in $>$ Table 1 , the HSH/US method used to formulate lipid nanocarriers resulted in a suitable way produce systems characterized by a mean diameter around $200 \mathrm{~nm}$ and a polydispersivity index around 0.30 . In particular, the latter parameter reflects the presence of homogeneous nanoparticles with minimum predisposition to aggregation [19]. The use of sesame oil instead of Miglyol 812 seems to not affect the dimension and the homogeneity of NLCs ( $\triangleright$ Table 1 ), while a different trend has been observed in relation to the drug recovery values. As expected, SLNs had a lower encapsulation efficiency compared to NLC-M and NLC-PLUS, which showed a drug recovery of $82.5 \%$ and $91.2 \%$, respectively. The best performance of NLC-PLUS is probably due to a high chemical affinity between SES and the sesame oil with the consequent drug concentration in the lipid matrix of the NLCs. A DSC analysis was performed to provide information about the lipid-solid state modification in nanoparticulate form and about SES accommodation into the core of the lipid matrix [20]. - Fig. 2 depicts the DSC profile of the pure materials, the empty nanoparticles (empty SLNs, empty NLC-M, and empty NLC-PLUS) and SES loaded nanoparticles (SLNs, NLC-M, and NLC-PLUS). The melting point peaks of the pure materials Lutrol, Compritol 888 ATO, and SES are at 54.24, 73.94, and $65.44^{\circ} \mathrm{C}$, respectively. All nanoparticles showed two melting point attributable to Lutrol and Compritol 888 ATO, with a slight shift (to $50-51^{\circ} \mathrm{C}$ and $71-72.5^{\circ} \mathrm{C}$, respectively) due to the structuring in the nanoparticulate form [21]. Furthermore, in the thermogram of all the loaded nanoparticles, the SES peak disappears. This indicates the complete solubilization of SES in the lipid matrix or the transformation of SES crystal to an amorphous form dispersed in the lipid matrix [20].

- Fig. 3 shows the cumulative amount $\left(Q_{24}\right)$ of SES permeated through human SCE membranes after $24 \mathrm{~h}$ of monitoring. Statistical analysis revealed a significant difference $(p<0.01)$ between the $Q_{24}$ value obtained for SES-GEL and the other formulations. Moreover, both NLC-M and NLC-PLUS showed $\mathrm{Q}_{24}$ values consider- 
- Table 1 Particle size, polydispersivity index (PDI), zeta potential, and drug recovery values of different lipid nanoparticle formulations.

\begin{tabular}{|l|l|l|l|l|}
\hline Sample & $\begin{array}{l}\text { Particle size } \\
\text { (nm, mean } \pm \text { SD) }\end{array}$ & $\begin{array}{l}\text { Polydispersivity index } \\
\text { (PDI, mean } \pm \text { SD) }\end{array}$ & $\begin{array}{l}\text { Zeta potential } \\
\text { (-mV, mean } \pm \text { SD) }\end{array}$ & $\begin{array}{l}\text { Drug recovery } \\
\text { (\%, mean } \pm \text { SD) }\end{array}$ \\
\hline Empty SLN & $206.1 \pm 18.5$ & $0.282 \pm 0.021$ & $-33.8 \pm 2.70$ & - \\
\hline SLN & $224.7 \pm 17.4$ & $0.287 \pm 0.040$ & $-35.0 \pm 2.41$ & $78.5 \pm 5.2$ \\
\hline Empty NLC-M & $169.1 \pm 20.2$ & $0.284 \pm 0.023$ & $-38.9 \pm 2.32$ & - \\
\hline NLC-M & $171.6 \pm 13.7$ & $0.277 \pm 0.032$ & $-36.1 \pm 2.14$ & $82.5 \pm 3.6$ \\
\hline Empty NLC-Plus & $169.0 \pm 10.1$ & $0.293 \pm 0.034$ & $-36.2 \pm 2.91$ & - \\
\hline NLC-Plus & $169.2 \pm 18.6$ & $0.317 \pm 0.043$ & $-38.0 \pm 2.62$ & $91.2 \pm 2.2$ \\
\hline
\end{tabular}

ably lower than SLNs $(p<0.01)$, this decreased permeation of SES when formulated in an NLC system could be explained by hypothesizing a drug accumulation in the horny layer. Both SLNs and NLCs have shown the peculiarity to reduce and/or suppress the permeation (transdermal delivery) through the skin while enhancing the penetration (dermal delivery) into the upper skin layers [22,23]. Geetha and coworkers have studied the anticancer effects of SES after incorporation into SLNs, demonstrating an interesting localized effect of the active compound in the skin [24].

In our study, the differences observed between SLNs and NLCS could be explained considering the key role of the oil phase being able, on the one hand, to retain the active compound inside the NLC matrix and, on the other hand, to release it in a controlled fashion.

Surprisingly, no significant differences $(p>0.05)$ were observed between the $\mathrm{Q}_{24}$ values obtained with NLC-M and NLCPLUS, although the latter showed the highest encapsulation efficiency (>90\%). This result seems to demonstrate that the oil phase characteristics did not affect the release of sesamol from the NLC matrix and therefore the chemical affinity between sesame oil and SES did not have an important role in this process.

Finally, we evaluated the antioxidant efficiency of all the different vehicles SLNs and NLCs. In particular, we assayed the capability of the empty SLNs and NLCs in comparison with SES alone and loaded formulations to prolong and/or to maintain the antioxidant activity of SES in the function of time using a standard ORAC assay. The results are shown in > Figs. 4-7. The FL signal disappears just to $2 \mathrm{~h}$ in the presence of a radical generator, such as AAPH. Indeed, it decreases in the presence of a sample endowed with a radical scavenging activity. The results showed that the empty SLNs were not active ( $\bullet$ Fig. 4 ), while the empty NLCs ( $\triangleright$ Figs. 5 and 6) showed an antioxidant activity under $19 \mathrm{~h}$. In particular, the presence of Miglyol 812 in empty NLC-M ( Fig. 5) gave a slight activity [25]. This increased activity in empty NLCPLUS ( $\bullet$ Fig. 6) is attributable to the intrinsic antioxidant activity of the sesame oil $[26,27]$. Nevertheless, also in this case, all the produced formulations were able to preserve and prolong the SES antioxidant activity until $40 \mathrm{~h}(\triangleright$ Fig. 7). In particular, the better result was obtained for the NLC-PLUS. In fact, the fluorescence value of NLC-M was $11400 \mathrm{~nm}$ ( $\triangleright$ Fig. 5), while that of NLC-PLUS was $17081 \mathrm{~nm}$ ( $\triangleright$ Fig. 6), both higher than the SLN value $(5581 \mathrm{~nm})(\triangleright$ Fig. 4).

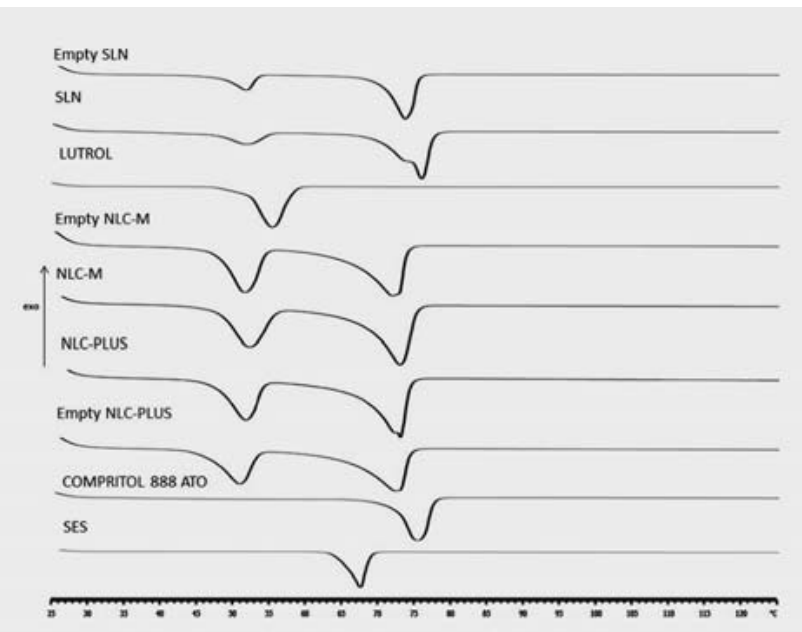

- Fig. 2 DSC thermogram scans of components and of empty or SES loaded lipid nanoparticles.

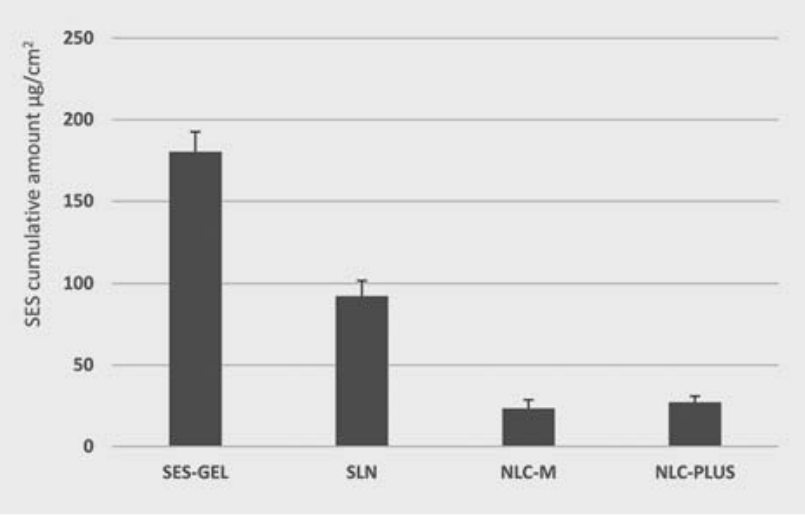

- Fig. 3 Cumulative amount $\left(Q_{24}\right)$ of SES permeated through SCE membranes during $24 \mathrm{~h}$ from SES-GEL, SLN, NLC-M, and NLC-PLUS. 


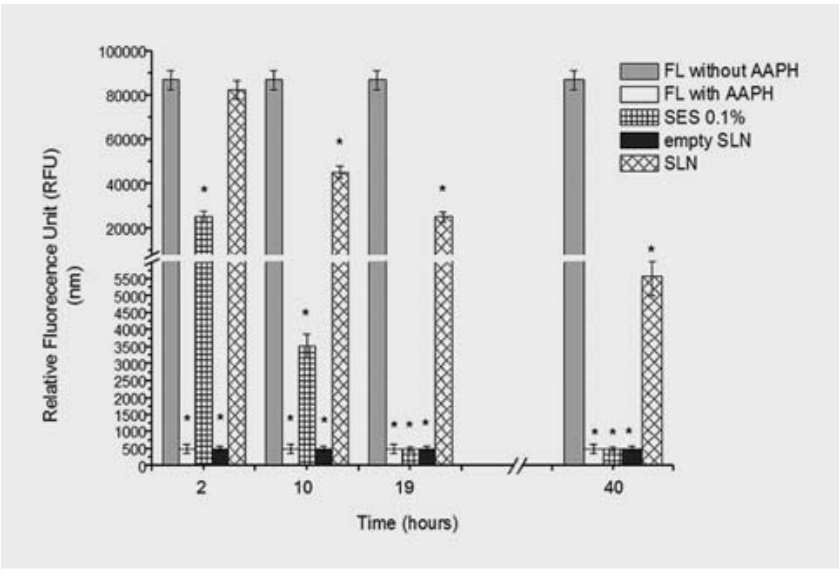

- Fig. 4 Antioxidant efficiency of SLN. Data represent the mean of three independent experiments \pm SD. ${ }^{*} P<0.05$.

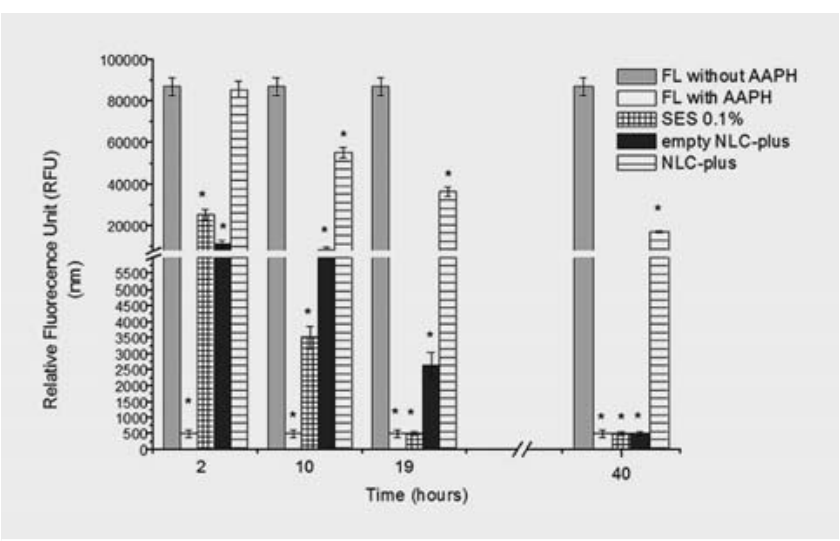

- Fig. 6 Antioxidant efficiency of NLC-PLUS. Data represent the mean of three independent experiments \pm SD. ${ }^{*} \mathrm{P}<0.05$.

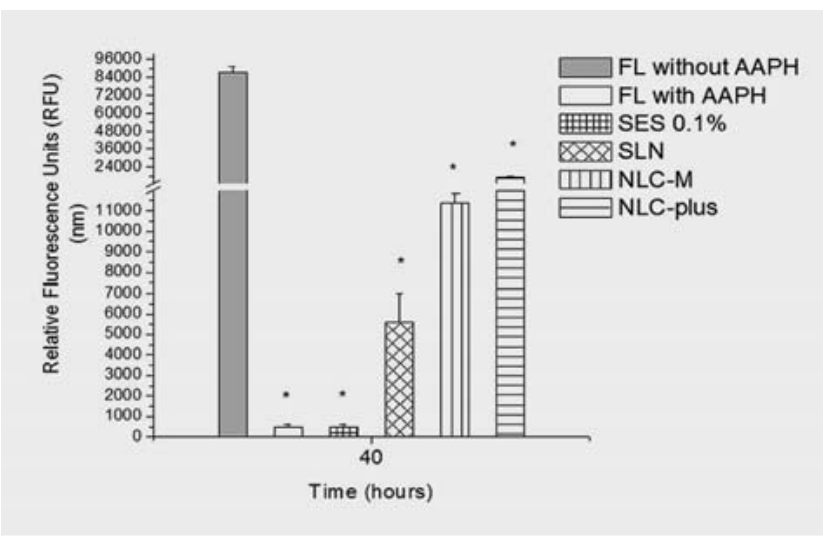

- Fig. 7 Antioxidant efficiency values at $40 \mathrm{~h}$ of all the formulations tested. Data represent the mean of three independent experiments \pm SD. ${ }^{*} P<0.05$.

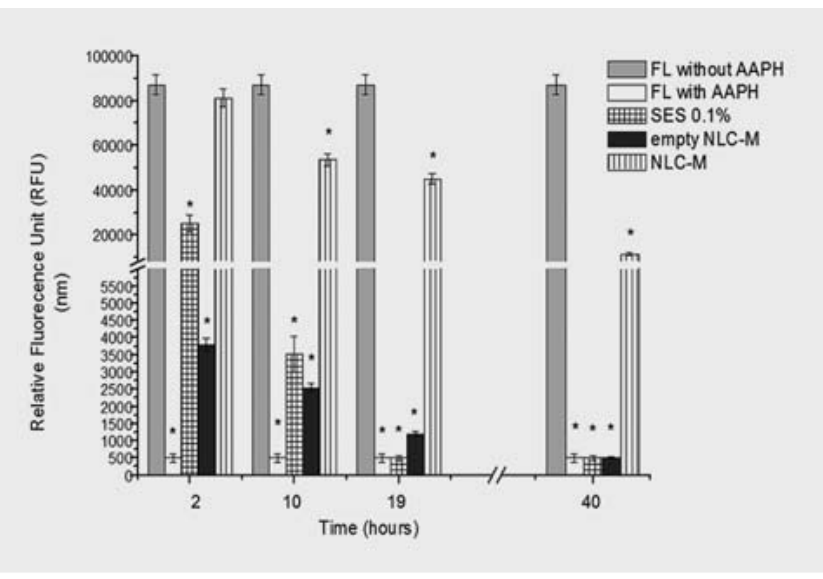

- Fig. 5 Antioxidant efficiency of NLC-M. Data represent the mean of three independent experiments \pm SD. ${ }^{*} P<0.05$.

\section{Materials and Methods}

\section{Materials}

SES (purity: 98\%) and sesame oil were purchased from Sigma-Aldrich srl. Compritol 888 ATO (glyceryl behenate, tribehenin), a mixture of mono-, di-, and triglycerides of behenic acid $\left(C_{22}\right)$, was provided by Gattefossè. Miglyol 812 (caprylic/capric triglycerides) was provided by Eigenmann\&Veronelli S. p. A. Lutrol F68 (Poloxamer 188) was obtained from BASF. Sodium polyacrylate was provided by Cognis S. p. A. Care Chemicals. All of the other chemicals and reagents were of the highest purity grade commercially available.

\section{Lipid nanoparticles production}

In this study, both NLCs and SLNs were formulated by using HSH and US methods $[9-13,18]$. In particular, SLNs were prepared as a reference vehicle to point out the advantages of SES incorporation in NLCs. In $>$ Table 2, the compositions of the SLNs and NLCs are reported. A weighted amount of Compritol $^{\circ} 888$ ATO was melted at $80^{\circ} \mathrm{C}$ adding SES to formulate SES loaded SLN. The melted lipid phase was dispersed in a hot $\left(80^{\circ} \mathrm{C}\right)$ aqueous solution containing Lutrol $^{\circ} \mathrm{F} 68$ by using a high-speed stirrer (UltraTurraxT25; IKA-Werke GmbH \& Co. KG); the obtained pre-emulsion was ultrasonified by using a UP $400 \mathrm{~S}$ Ultraschallprozessor (Dr. Hielscher $\mathrm{GmbH}$ ), maintaining the temperature at least $5^{\circ} \mathrm{C}$ above the lipid melting point. Then, the obtained hot dispersions were cooled in an ice bath under high-speed homogenization (UltraTurrax T25; IKA-Werke GmbH \& Co. KG) in order to solidify the lipid matrix and to form the SLNs. Empty SLNs were obtained following the previous protocol but without the addition of SES to the solid lipid. In this study, we evaluated two different kinds of NLCs whose composition is reported in $>$ Table 2 . NLC-M was formulated adding, at $80^{\circ} \mathrm{C}$, a weighted amount of SES to Miglyol 812 and then to the melted solid lipid Compritol 888. The obtained lipid phase was dispersed in a hot $\left(80^{\circ} \mathrm{C}\right)$ aqueous solution containing Lutrol ${ }^{\circ} \mathrm{F} 68$ and then the protocol followed the steps described for SLN preparation. NLC-PLUS was formulated using a weighted amount of ses- 
- Table 2 Composition (\% w/w) of NLC- and SLN-based formulations.

\begin{tabular}{|c|c|c|c|c|c|c|}
\hline \multirow[t]{2}{*}{ Constituents } & \multicolumn{6}{|c|}{ Formulation code } \\
\hline & Empty SLN & SLN & Empty NLC-M & NLC-M & Empty NLC-Plus & NLC-Plus \\
\hline Sesamol & - & 0.10 & - & 0.04 & - & 0.04 \\
\hline Sesame oil & - & - & - & - & 0.21 & 0.21 \\
\hline Compritol 888 ATO & 0.79 & 0.79 & 1.12 & 1.12 & 1.12 & 1.12 \\
\hline Miglyol 812 & - & - & 0.21 & 0.21 & - & - \\
\hline Lutrol F68 & 0.30 & 0.30 & 0.63 & 0.63 & 0.63 & 0.63 \\
\hline Water & 98.91 & 98.81 & 98.04 & 98.00 & 98.04 & 98.00 \\
\hline
\end{tabular}

ame oil instead of Mygliol 812 (see - Table 2). Blank NLC-M and NLC-PLUS were formulated to be used as reference vehicles.

\section{Particle size distribution and zeta potential measurements}

Photon correlation spectroscopy (PCS) was used to measure mean particle size and the zeta potential of the lipid dispersions. A Zetamaster (Malvern Instrument Ltd.), equipped with a solid state laser having a nominal power of $4.5 \mathrm{~mW}$ with a maximum output of $5 \mathrm{~mW} 670 \mathrm{~nm}$ and a $90^{\circ}$ scattering angle at $20 \pm 0.20^{\circ} \mathrm{C}$, was employed. Samples ( $10 \mu \mathrm{L}$ of the suspension) were diluted with $2 \mathrm{~mL}$ of deionized water previously filtered through a 0.2- $\mu \mathrm{m}$ Acrodisc LC 13 PVDF filter (Pall-Gelman Laboratory). During the experiment, the refractive index of the samples always matched the liquid (toluene) to avoid stray light. The electrophoretic mobility was calculated using Smoluchowski's equation (Eq. 1) to determine the zeta $(\xi)$ potential value.

$\mathrm{v}=\left(\frac{\varepsilon \cdot \mathrm{E}}{\eta}\right) \xi$

where $v$ is the measured electrophoretic velocity, $\eta$ is the viscosity, $\varepsilon$ is the electrical permittivity of the electrolytic solution, and $E$ is the electric field. The accuracy was $0.12 \mu \mathrm{m} \mathrm{cm} / \mathrm{V}$ s for the aqueous systems. Samples were suspended in distilled water and the measures were recorded at $25^{\circ} \mathrm{C}$.

\section{Determination of drug loading}

The percentage of SES entrapped in the lipid matrix was determined as follows: a fixed amount of SLN or NLC dispersion was filtered using a Pellicon XL tangential ultrafiltration system (Millipore) equipped with a polyethersulfone Biomax 1000 membrane (Millipore) with a $1000000 \mathrm{Da}$ molecular weight cutoff. An amount of retained material was freeze-dried, dissolved in dichloromethane, and analyzed by UV spectrophotometry at $290 \mathrm{~nm}$ (Lambda 52, PerkinElmer). Calibration curves for the validated UV assays of SES were performed on six solutions in the concentration range of $10-100 \mu \mathrm{g} / \mathrm{mL}$. The correlation coefficient was $>0.99$.

Each point represented the average of three measurements, and the error was calculated as the standard deviation ( \pm SD).
SES incorporation efficiency was expressed as active recovery and calculated using equation 2 (Eq. 2 ):

Drug recovery $(\%)=\frac{\text { Mass of SES in nanoparticles }}{\text { Mass of SES fed to the system }} \times 100$

Possible lipid interferences during UV determination of SES were also investigated by comparing the two standard curves of the substance alone and in the presence of lipids. The differences observed between the standard curves were within the experimental error, thus inferring that no lipid interference occurred (data not shown).

\section{Differential scanning calorimetry}

Raw materials and unloaded and loaded nanoparticles were analyzed by differential scanning calorimetry on an indium calibrated Mettler Toledo DSC 822e (Mettler Toledo). Thermograms were recorded by placing accurately given quantities $(6-7 \mathrm{mg}$ weighed with a microbalance MTS Mettler Toledo) of each sample in a 40$\mu \mathrm{L}$ aluminium pan that was sealed and pierced. The sample was scanned $\left(10^{\circ} \mathrm{C} / \mathrm{min}\right)$ between $25-125^{\circ} \mathrm{C}$. From the heated cycle, the melting temperature $\mathrm{Tm}$ and heats of fusion $(\Delta \mathrm{Hm})$ were measured.

\section{In vitro percutaneous absorption studies}

Gel preparation: For in vitro studies, SLNs, NLC-M, and NLC-PLUS were formulated into hydrogel using sodium polyacrylate (1\% w/ w) as a thickener agent. Furthermore, an SES-based hydrogel (SES-GEL) was formulated and used as a reference vehicle. The final concentration of SES in the hydrogel formulations was $0.1 \%$ $\mathrm{w} / \mathrm{w}$. All the formulations were stored at $4^{\circ} \mathrm{C}$ before use.

Skin membrane preparation: Samples of adult human skin (mean age $36 \pm 8$ years) were obtained from breast reduction operations. Subcutaneous fat was carefully trimmed and the skin was immersed in distilled water at $60 \pm 1.0^{\circ} \mathrm{C}$ for 2 min [28], after which SCE was removed from the dermis using a dull scalpel blade. Epidermal membranes were dried in a desiccator at $25 \%$ relative humidity. The dried samples were wrapped in aluminum foil and stored at $4 \pm 1.0^{\circ} \mathrm{C}$ until use. Previous research work demonstrated the maintenance of SC barrier characteristics after storage under the reported conditions [29]. Besides, preliminary experiments were carried out in order to assess the barrier integrity 
of SCE samples by measuring the in vitro permeability of $\left[{ }^{3} \mathrm{H}\right]$ water through the membranes using the Franz cell method described below. The value of the calculated permeability coefficient $(\mathrm{Pm})$ for $\left[{ }^{3} \mathrm{H}\right]$ water agreed well with those previously reported [30].

\section{In vitro skin permeation experiments}

Samples of dried SCE were rehydrated by immersion in distilled water at room temperature for $1 \mathrm{~h}$ before being mounted in Franz-type diffusion cells supplied by LGA. The exposed skin surface area was $0.75 \mathrm{~cm}^{2}$ and the receiver compartment volume was $4.5 \mathrm{~mL}$.

The receptor compartment was filled with a water-ethanol solution (50:50) to allow the establishment of the sink conditions and to sustain permeant solubilization [31]. Furthermore, it was stirred at $500 \mathrm{rpm}$ and thermostated at $35 \pm 1.0^{\circ} \mathrm{C}$ throughout all of the experiments [12]. A weighted amount of SLNs, NLC-M, NLCPLUS, and SES-GEL was placed on the skin surface in the donor compartment and the latter was covered with Parafilm ${ }^{\odot}$. Each experiment was run in duplicate for $24 \mathrm{~h}$ using three different donors $(n=6)$. The samples were analyzed for SES content by HPLC as described below. The results are expressed as the cumulative amount of SES permeating the SCE membranes after $24 \mathrm{~h}$.

\section{HPLC}

The HPLC apparatus consisted of a Shimadzu LC10 AT Vp equipped with a 20- $\mu \mathrm{L}$ loop injector and an SPD-M $10 \mathrm{~A} \mathrm{Vp}$ Shimadzu photodiode array UV detector. Chromatography was performed using an Ascentis Express C18 column (particle size, $2.7 \mu \mathrm{m} ; 150 \times 4.6 \mathrm{~mm}$ i.d.; Supelco, Sigma-Aldrich). The mobile phase was composed of water and methanol (30:70) and the detection was effected at $290 \mathrm{~nm}$. The flow rate was set at $0.8 \mathrm{~mL}$ min and the retention time was 5.9 minutes.

\section{Antioxidant efficiency}

A modified ORAC assay was used to qualitatively determine the antioxidant activity of SLNs, NLC-M, and NLC-PLUS [32]. SES and empty and loaded vehicles $(25 \mu \mathrm{L})$ were placed in 96-well tissue culture plates. $150 \mu \mathrm{L}$ of FL ( $10 \mathrm{nM})$ was used as the probe to assess the antioxidant activity. $25 \mu \mathrm{L}$ of the water-soluble azo-compound AAPH (100 mM) was used as a radical initiator to generate free radicals at a constant rate. A positive control (FL solution containing $A A P H)$, a negative control (FL solution containing no AAPH), SES (0.1\%), and unloaded and loaded SLNs and NLCs in PBS were run simultaneously. A timer was started upon introduction of the free radical generator and the plate was stored in the dark at $37^{\circ} \mathrm{C}$. At each specified time point, the fluorescence of the solution was measured (excitation $492 \mathrm{~nm}$, emission $535 \mathrm{~nm}$ ) using a Wallac 1420 Victor 3 96-well plate reader (Perkin Elmer) fluorimeter and plotted as a function of time with Origin ${ }^{\circledR} 7$ software (Origin Lab Corporation). The Y axis graph was split as follow: 6000 to 8000 RFU ( $\triangleright$ Figs. 4-6) and from 12000 to 13000 RFU ( $\vee$ Fig. 7).

\section{Statistical analysis}

Statistical analysis of the data was performed using the Student's t-test. A probability ( $p$ ) of less than 0.05 is considered significant in this study.

\section{Conflict of Interest}

The authors declare no conflict of interest.

References

[1] Kaur IP, Saini A. Sesamol exhibits antimutagenic activity against oxygen species mediated mutagenicity. Mutat Res 2000; 470: 71-76

[2] Kapadia G], Azuine MA, Tokuda H, Takasaki M, Mukainaka T, Konoshima T, Nishino H. Chemopreventive effect of resveratrol, sesamol, sesame oil and sunflower oil in the Epstein-Barr virus early antigen activation assay and the mouse skin two-stage carcinogenesis. Pharmacol Res 2002; 45: 499-505

[3] Shimizu S, Fujii G, Takahashi M, Nakanishi R, Komiya M, Shimura M, Noma N, Onuma W, Terasaki M, Yano T, Mutoh M. Sesamol suppresses cyclooxygenase-2 transcriptional activity in colon cancer cells and modifies intestinal polyp development in Apc (Min/+) mice. J Clin Biochem Nutr 2014; 54: 95-101

[4] Sharma S, Kaur IP. Development and evaluation of sesamol as an antiaging agent. Int J Dermatol 2006; 45: 200-208

[5] Baek S. Sesamol decreases melanin biosynthesis in melanocyte cells and zebrafish: Possible involvement of MITF via the intracellular CAMP and p 38/JNK signaling pathways. Exp Dermatol 2015; 24: 761-766

[6] Srisayam M, Weerapreeyakul N, Barusrux S, Kanokmedhakul K. Antioxidant, antimelanogenic, and skin-protective effect of sesamol. J Cosmet Sci 2014; 65: 69-79

[7] Müller RH, Radtke M, Wissing SA. Nanostructured lipid matrices for improved microencapsulation of drugs. Int J Pharm 2002; 242: 121-128

[8] Puglia C, Bonina F. Lipid nanoparticles as novel delivery systems for cosmetics and dermal pharmaceuticals. Expert Opin Drug Deliv 2012; 9: 429-441

[9] Puglia C, Damiani E, Offerta A, Rizza L, Tirendi GG, Tarico MS, Curreri S, Bonina F, Perrotta RE. Evaluation of nanostructured lipid carriers (NLC) and nanoemulsions as carriers for UV-filters: characterization, in vitro penetration and photostability studies. Eur J Pharm Sci 2014; 51: 211217

[10] Puglia C, Sarpietro MG, Bonina F, Castelli F, Zammataro M, Chiechio S. Development, characterization, and in vitro and in vivo evaluation of benzocaine- and lidocaine-loaded nanostructrured lipid carriers. J Pharm Sci 2011; 100: 1892-1899

[11] Pinto MF, Moura CC, Nunes C, Segundo MA, Costa Lima SA, Reis S. A new topical formulation for psoriasis: development of methotrexate-loaded nanostructured lipid carriers. Int J Pharm 2014; 477: 519-526

[12] Ricci M, Puglia C, Bonina F, Di Giovanni C, Giovagnoli S, Rossi C. Evaluation of indomethacin percutaneous absorption from nanostructured lipid carriers (NLC): in vitro and in vivo studies. J Pharm Sci 2005; 94: 11491159

[13] Müller RH, Radtke M, Wissing SA. Solid lipid nanoparticles (SLN) and nanostructured lipid carriers (NLC) in cosmetic and dermatological preparations. Adv Drug Deliv Rev 2002; 54: S131-S155

[14] Wan Y, Li H, Fu G, Chen X, Chen F, Xie M. The relationship of antioxidant components and antioxidant activity of sesame seed oil. J Sci Food Agric 2015; 95: 2571-2578

[15] Yoshida H, Shigezaki J, Takagi S, Kajimoto G. Variation in the composition of various acyl lipid, tocopherols and lignans in sesame seed oil roasted in a microwave oven. J Sci Food Agric 1995; 68: 407-415 
[16] Namiki M, Fukuda Y, Takei Y, Namiki K, Koizumi Y. Changes in functional factors of sesame seed and oil during various types of processing. ACS Symp Ser 2002; 816: 85-104

[17] Yoshida H, Takagi S. Antioxidative effects of sesamol and tocopherols at various concentrations in oils during microwave heating. J Sci Food Agric 1999; 79: 220-226

[18] Puglia C, Offerta A, Rizza L, Zingale G, Bonina F, Ronsisvalle S. Optimization of curcumin loaded lipid nanoparticles formulated using high shear homogenization (HSH) and ultrasonication (US) methods. J Nanosci Nanotechnol 2013; 13: 6888-6893

[19] Mitri K, Shegokar R, Gohla S, Anselmi C, Müller RH. Lipid nanocarriers for dermal delivery of lutein: preparation, characterization, stability and performance. Int J Pharm 2011; 414: 267-275

[20] Catenacci L, Sorrenti M, Bruni G, Bonferoni MC, Sandri G, Bettinetti G. Characterization of silver sulfadiazine-loaded solid lipid nanoparticles by thermal analysis. J Therm Anal Calorim 2013; 111: 2149-2215

[21] Dan S, Ng WK, Tan RBH. Are nanostructured lipid carriers (NLCs) better than solid lipid nanoparticles (SLNs): development, characterizations and comparative evaluations of clotrimazole-loaded SLNs and NLCs? Eur J Pharm Sci 2012; 47: 139-151

[22] Lombardi Borgia S, Regehly M, Sivaramakrishnan R, Mehnert W, Korting HC, Danker K, Röder B, Kramer KD, Schäfer-Korting M. Lipid nanoparticles for skin penetration enhancement-correlation to drug localization within the particle matrix as determined by fluorescence and parelectric spectroscopy. J Control Release 2005; 110: 151-163

[23] Chen H, Chang X, Du D, Liu W, Liu J, Weng T, Yang Y, Xu H, Yang X. Podophyllotoxin-loaded solid lipid nanoparticles for epidermal targeting. J Control Release 2006; 110: 296-306
[24] Geetha T, Kapila M, Prakash O, Deol PK, Kakkar V, Kaur IP. Sesamolloaded solid lipid nanoparticles for treatment of skin cancer. J Drug Target 2015; 23: 159-169

[25] Silvestre MP, Chaiyasit W, Brannan RG, McClements DJ, Decker EA. Ability of surfactant head group size to alter lipid and antioxidant oxidation in oil-in-water emulsions. J Agric Food Chem 2000; 48: 2057-2061

[26] Carvalho RHR, Galvao EL, Barros JAC, Conceicao MM, Sousa EMBD. Extraction, fatty acid profile and antioxidant activity of sesame extract (Sesamum Indicum L.). Braz J Chem Eng 2012; 29: 409-420

[27] Bopitiya D, Madhujith T. Antioxidant activity and total phenolic content of sesame (Sesamum indicum L.) seed oil. Trop Agric Res 2013; 24: 296302

[28] Kligman AM, Christophers E. Preparation of isolated sheets of human stratum corneum. Arch Dermatol 1963; 88: 702-705

[29] Swarbrick J, Lee G, Brom J. Drug permeation through human skin. I. Effects of storage conditions of skin. J Invest Dermatol 1982; 78: 63-66

[30] Bronaugh RL, Stewart RF, Simon M. Methods for in vitro percutaneous absorption studies. VII: Use of excised human skin. J Pharm Sci 1986; 75: 1094-1097

[31] Toitou E, Fabin B. Altered skin permeation of highly lipophilic molecule: Tetrahydrocannabinol. Int J Pharm 1988; 43: 17-22

[32] Merrell JG, McLaughlin SW, Tie L, Laurencin CT, Chen AF, Nair LS. Curcumin-loaded poly(epsilon-caprolactone) nanofibres: diabetic wound dressing with anti-oxidant and anti-inflammatory properties. Clin Exp Pharmacol Physiol 2009; 36: 1149-1156 